\title{
Electroacupuncture treatment contributes to the downregulation of neuronal nitric oxide synthase and motoneuron death in injured spinal cords following root avulsion of the brachial plexus
}

\author{
HAOXUAN LUO ${ }^{1}$, XIAO CHENG ${ }^{2}$, YING TANG ${ }^{1}$, ZEMIN LING $^{1}$ and LIHUA ZHOU ${ }^{1}$ \\ ${ }^{1}$ Department of Anatomy, Zhongshan School of Medicine, Sun Yat-sen University, Guangzhou, Guangdong 510080; \\ ${ }^{2}$ Encephalopathy Center, Guangdong Hospital of Traditional Chinese Medicine, Guangzhou, Guangdong 510120, P.R. China
}

Received October 02, 2013; Accepted November 19, 2013

DOI: $10.3892 /$ br.2013.212

\begin{abstract}
This study was performed in order to investigate the effect of electroacupuncture (EA) on motoneurons and the expression of neuronal nitric oxide synthase (nNOS) following brachial plexus root avulsion (BPRA). A total of 40 female Sprague-Dawley rats underwent BPRA (5th cervical-1st thoracic) and were randomly divided into the avulsion plus EA stimulation $(\mathrm{AV}+\mathrm{EA})$ and $\mathrm{AV}$ groups. The AV+EA group received a continuous $20-\mathrm{Hz}$ asymmetric bidirectional disperse-dense wave at the acupuncture points (acupoints) of Dazhui (DU4) and Shousanli (LI10) for $15 \mathrm{~min}$ on alternate days until the animals were sacrificed, at 1, 2, 3 and 6 weeks. The AV group received no treatment. The cryostat sections of the 7 th cervical segments were prepared and stained with neuronal nitric oxide synthase nicotinamide adenine dinucleotide phosphate diaphorase (NADPH-d) and histochemically stained and counterstained with neutral red (NR). The number of nNOS-positive motoneurons on the lesion side and survived motoneurons on both sides of the 7 th cervical segments were blindly counted and compared between the two groups. The results demonstrated that the number of nNOS-positive motoneurons was significantly lower in the AV+EA group compared with that in the AV group and the percentage of survived motoneurons was significantly higher compared with that of the AV group at 2 and 3 weeks. However, the number of nNOS-positive motoneurons and the percentage of survived motoneurons were not significantly
\end{abstract}

Correspondence to: Professor Lihua Zhou, Department of Anatomy, Zhongshan School of Medicine, Sun Yat-sen University, 74 Zhongshan Er Road, Guangzhou, Guangdong 510080, P.R. China E-mail: zhoulih@mail.sysu.edu.cn

Dr Xiao Cheng, Enchephalopathy Center, Guangdong Hospital of Traditional Chinese Medicine, 111 Dade Road, Guangzhou, Guangdong 510120, P.R. China

E-mail: chengxiaolucky@126.com

Key words: electroacupuncture, brachial plexus root avulsion, motoneuron, neuronal nitric oxide synthase different between the two groups at 1 and 6 weeks. These results indicated that, during the early period after BPRA, EA stimulation at the acupoints of Dazhui (DU4) and Shousanli (LI10) may significantly reduce the number of nNOS-positive motoneurons and protect against motoneuron death.

\section{Introduction}

Brachial plexus root avulsion (BPRA) is a severe peripheral nerve injury (PNI) which leads to an almost complete loss of sensory and motor function in the upper limb, accompanied by a characteristic constant crushing and intermittent shooting pain that is often intractable. BPRA generally occurs as a result of traffic accidents or as part of the birth injury syndrome (1).

Previous studies demonstrated that BPRA induced de novo expression of neuronal nitric oxide synthase (nNOS) in motoneurons, which has been considered to be closely associated with motoneuron degeneration (2-6). In previous studies by our research group, we also observed that the time course and density of avulsion (AV)-induced nNOS expression were correlated with the severity of motoneuron death (7). Additionally, the downregulation of existing nNOS expression through the implantation of a peripheral nerve graft or the application of exogenous glial cell line-derived neurotrophic factor (GDNF) or brain-derived neurotrophic factor (BDNF) blocks may protect the injured motoneurons from death. These findings indicated that injury-induced nNOS exerted a neurotoxic effect in motoneuron survival following root AV. Therefore, a selective blockade of nNOS production may be a useful approach for reducing motoneuron death. Pharmacological inhibitors of NOS have been reported previously in PNI models (8).

Previous studies have mainly focused on increasing the number of survived motoneurons by downregulating the expression of nNOS and, despite a significant progress, there were certain limitations. Chai et al (9) reported that, with the implantation of a peripheral nerve graft, the survival rate of motoneurons was increased from 65 to $90 \%$ and from 39 to $80 \%$ at 3 and 6 weeks following BPRA, respectively; furthermore, the expression of nNOS was significantly inhibited. However, that procedure required high precision and high-level operators; additionally, it increased the possibility 
of infection. Su et al (10) demonstrated that the application of lithium chloride for $>4$ weeks following replantation protected motoneurons by decreasing the number of microglia and macrophages. However, the administration of lithium chloride is accompanied by side effects such as dizziness, nausea, vomiting, diarrhea, convulsions and comas. During the experiment, we observed that the application of lithium chloride led to distortion of the animals' bodies, with muscle rigidity accompanied by significant pain. Therefore, the application of lithium chloride has limitations. Furthermore, the application of antioxidants and neurotropic factors to protect injured motoneurons was also accompanied by side effects and limitations; therefore, they may not be widely utilized. Consequently, traditional Chinese medicine (TCM) was considered to be a viable option, due to its minimal side effects, simplicity and safety.

TCM is a significant part of Chinese culture; acupuncture and electroacupuncture (EA), which combines acupuncture with modern medicine, were shown to exert positive effects on the treatment of PNI $(11,12)$. According to TCM, acupuncture meridians run Qi and blood through unique channels, which have their corresponding organs and organizations (13). Furthermore, EA therapy was shown to exert a positive effect on neural and functional recovery following spinal cord injury (14). In a rat sciatic nerve crush model, EA exerted a positive effect on motoneuron recovery and was efficient in treating pain symptoms that had developed during targeted re-innervation (11). Furthermore, in a rat thigh crush model, EA with direct current (DCEA) contributed to the regeneration of the peripheral nerves $(15,16)$. EA may have several therapeutic roles through different underlying mechanisms. EA may regulate the homeostasis of the cardiovascular system; correct $\mathrm{pH}$ imbalances caused by chronic hypoxia by adjusting the levels of endothelin-1 (ET-1) and endothelial nitric oxide synthase (eNOS) (17); and relieve mechanical allodynia in neuropathic rats through inhibiting nNOS expression in the spinal cord (18). High-frequency EA may also effectively correct the behavioral disorders of rats with Parkinson's disease (PD) by reducing the amino acid (GABA) levels in the ventral midbrain (19). In a chronic-constriction injury of the rat sciatic nerve model, EA was shown to alter pain behavior through its antinociceptive effects (20). The studies mentioned previously demonstrated that EA exerted curative effects on disorders of the nervous system.

BPRA is a type of PNI that fits the 'flaccidity pattern' according to TCM; this pattern is associated with several symptoms, such as limb tendon reflex retardation, weakness and lack of free movements, which may lead to muscle flaccidity. According to the principles of TCM, the large intestine meridian (LI), stomach meridian (ST) and Du meridian (DU), are selected for acupuncture in the treatment of such disorders, due to the functions of the Yangming meridian, which may cure nerve and muscle dysfunctions due to its abundance of Qi and blood. As a consequence, the upper and lower extremity meridian acupoints were selected, as they could dredge meridians and activate collaterals, tonify Qi and replenish blood, which was described by the phrase 'Yangming is chosen to cure flaccidity pattern only' in TCM; the DU acupoints were selected as they may relax sinews and activate collaterals by adjusting the Yin and Yang of organs, activating blood and moving Qi $(21,22)$.
However, there are currently no studies available on whether EA exerts an effect on BPRA.

Therefore, the acupoints of Dazhui (DU4) and Shousanli (LI10) were selected to treat rats with BPRA in this study. The number of nNOS-positive motoneurons and the percentage of survived motoneurons were used to evaluate the effects of EA on BPRA. With this study, we aimed to provide experimental data on applying EA as a clinical method in the treatment of BPRA.

\section{Materials and methods}

Animals. A total of 40 female Sprague-Dawley rats, weighing 180-220 g, were provided by the Laboratory Animal Center of Sun Yat-sen University (Guangzhou, China) [certification no. SCXK (Yue) 2011-0029]. The animals were housed under a 12-h light/dark cycle, with free access to food and water.

All experimental procedures were approved by the Committee on the Use of Live Animals for Teaching and Research of Sun Yat-sen University.

Establishment of BPRA models. The operation steps were based on our previous study (8). Following anesthesia with injection of $10 \%$ chloral hydrate $(3.5 \mathrm{ml} / \mathrm{kg})$ intraperitoneally, the rats were laid under a Shangyiguang SXP-1C surgical microscope (Shanghai Medical Instruments Co., Ltd., Shanghai, China). The skin was incised along the lower border of the right clavicle and a layered dissection was performed to expose the right brachial plexus in the axillary cavity to the intervertebral foramina. After the nerve roots contributing to the brachial plexus were identified and individually separated to the lateral side of the corresponding intervertebral foramina, they were tightly clamped by a microscopic hemostat near the intervertebral foramina. Finally, the roots were successively avulsed with the hemostat. When the entire root and its dorsal root ganglion were clearly identified under the microscope, the success of the surgery was confirmed. The skin was subsequently sutured and the animals were kept in warm chambers and allowed to recover from the anaesthesia prior to returning to their cages. The rats which had been successfully operated on were randomly divided into the AV and AV+EA groups.

Fixing and EA stimulation. The locations of the acupoints of DU4 and LI10 were based on anatomical landmarks and finger cun (proporational ruler). Their locations are summarized in Table I and illustrated in Fig. 1 (21,23-25).

The BPRA model rats received EA treatment, initiated on the day following the operation and administered every other day, until they were sacrificed, at 1,2, 3 and 6 weeks. In order to mimic the clinical treatment procedures, the experiment required that the rats were awake prior to and following the EA procedure. To avoid any damage or stress response potentially caused by a rigid fixing method, a flexible fixing method was applied. The rats were fixed on a soft-type rat-fixing instrument, which was developed by Wenzhou Medical College (national patent no. ZL 2011.2.2216075.0) (Fig. 1). Following a local alcohol swab, a 0.5-cun Hwato SDZ-2 Electronic Acupuncture needle (Suzhou Medical Appliance Factory, Suzhou, China) was vertically inserted into Dazhui (DU4) and obliquely into Shousanli (LI10), $5 \mathrm{~mm}$ below the 
Table I. Location and needling methods of Dazhui (DU4), Shousanli (LI10) and Quchi (LI11).

\begin{tabular}{|c|c|c|c|}
\hline Acupoints & Method of location & Anatomic locations & Methods of needling \\
\hline Dazhui (DU4) & Anatomic landmark & $\begin{array}{l}\text { Fossa below the spinal process } \\
\text { of the } 7 \text { th cervical vertebra, } \\
\text { midline of the back }\end{array}$ & $5-\mathrm{mm}$ insertion vertically \\
\hline Shousanli (LI10) & Proportional ruler & $\begin{array}{c}\text { Folds formed by the extensor muscles } \\
\text { and } \sim 10 \mathrm{~mm} \text { below the Quchi (LI11), } \\
\text { or between the radial side of the } \\
\text { wrist extensor digitorum }\end{array}$ & 5-mm insertion obliquely \\
\hline Quchi (LI11) & Anatomic landmark & $\begin{array}{l}\text { Fossa on the lateral } \\
\text { anterior side of the joint of } \\
\text { the proximal radius }\end{array}$ & Not used in this study \\
\hline
\end{tabular}

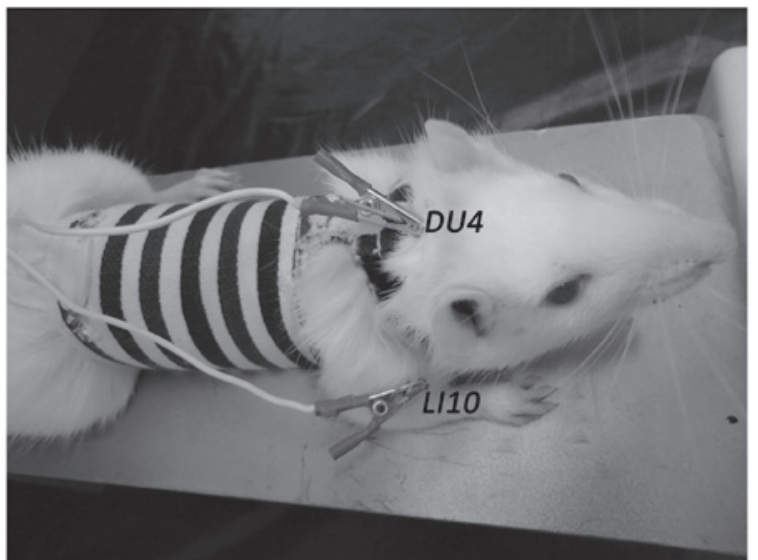

Figure 1. Fixing method and electroacupuncture stimulation. The rats were fixed by a new soft-type fixing instrument improved by Wenzhou Medical College. With this instrument, the damage caused by the traditional hard-type fixing method may be avoided. Furthermore, the rats were awake and made to feel comfortable during the treatment procedure.

skin; the acupuncture trocar was used if necessary. Finally, the needles were connected with the electrodes of the Hwato SDZ-2 Electronic Acupuncture Treatment Instrument with a frequency of $20 \mathrm{~Hz}$ and a continuous asymmetric bidirectional disperse-dense wave was applied for $15 \mathrm{~min}$.

Spinal cord tissue processing. The steps of spinal cord tissue processing were based on our previous studies $(26,7)$. The rats were sacrificed at 1,2,3 and 6 weeks. Following anesthesia with injection of $10 \%$ chloral hydrate $(3.5 \mathrm{ml} / \mathrm{kg})$ intraperitoneally, the thoracic cavity was opened, the ascending aorta was intubated via the left ventricle and perfused with a solution of cold $0.9 \%$ saline until blanching of the liver. Subsequently, the $0.9 \%$ saline was replaced by cold $4 \%$ paraformaldehyde in phosphate buffer (PB) (pH 7.4) until the liver and muscles were stiff. The 7 th segment of the cervical spinal cord was removed from its midpoints of proximal and distal nerve roots, placed in cold $4 \%$ paraformaldehyde in $\mathrm{PB}(\mathrm{pH} 7.4)$ for $4 \mathrm{~h}$ and then transferred to $30 \%$ sucrose solution in a $4^{\circ} \mathrm{C}$ refrigerator overnight, until it sunk to the bottom of the centrifuge tube. The 7 th cervical spinal cord tissues were sliced in the horizontal plane in $35-\mu \mathrm{m}$ sections by a semiconductor cryostat microtome (Leica Biosystems, Mannheim, Germany). Finally, every third tissue section was obtained and placed in $0.01 \mathrm{~mol} / \mathrm{l} \mathrm{PB}$ (pH 8.0).

Histochemistry stain for nNOS and neutral red (NR) counterstain for motoneurons. The staining steps were based on our previous studies $(7,25,26)$. There were $10-15$ sections and $100 \mu \mathrm{l}$ nicotinamide adenine dinucleotide phosphate diaphorase (NADPH-d) stain solution per well of a 96-well plate. The solution was composed of $0.1 \mathrm{mg}$ NADPH-d, $3 \mu \mathrm{l}$ Triton-100 and $\sim 0.25 \mathrm{mg}$ nitro blue tetrazolium, with the addition of $0.05 \mathrm{~mol} / \mathrm{l}$ Tris-buffer to a final volume of $100 \mu \mathrm{l}$. The 96-well plate was transferred to an incubator with a temperature of $37^{\circ} \mathrm{C}$ for 1 to $2 \mathrm{~h}$, until the blue nNOS-positive motoneurons were visible around the central canal of the spinal cord. Subsequently, the sections were pasted on the slides and rinsed three times with $0.1 \mathrm{~mol} / 1 \mathrm{~PB}(\mathrm{pH} \mathrm{8.0)}$; the slides were then counterstained with NR for 4 to $6 \mathrm{~h}$, dehydrated by 70, 80, 90 and $95 \%$ alcohol and $100 \%$ ethanol, dealcoholized by immersion in xylene three times $(10 \mathrm{~min}$ per time) and finally covered by coverslips and sealed with neutral gum.

Cell counting of motoneurons. The motoneurons in the 7 th cervical spinal cord segment were counted blindly on lesion and control sides in all the sections stained with NADPH-d and counterstained with NR, as previously described (7). On the contralateral side, the number of motoneurons served as normal control (expression of $100 \%$ ). On the side of the lesion, the dark blue-stained nNOS-positive motoneurons were blindly counted. The number of survived motoneurons included NADPH-d-positive and -negative motoneurons and it was expressed as a percentage of the number of motoneurons on the contralateral side of the same 7 th cervical section. The number of nNOS-positive motoneurons only included NADPH-d-positive motoneurons and it was expressed as a percentage of the number of motoneurons on the contralateral side of the same 7 th cervical section. The formulas for the calculation of the rate of nNOS positivity and the survival of motoneurons are summarized in Table II. The nNOS-positive motoneurons and the percentage of survived motoneurons were compared among these groups.

Statistical analysis. Data were analyzed by Statistical Product and Service Solutions (SPSS) software, with an 
Table II. Calculation of neuronal nitric oxide synthase (nNOS)-positive motoneurons and percentage of survived motoneurons.

Term

nNOS-positive motoneurons $(\%)$

Percentage of survived motoneurons $(\%)$
Formula
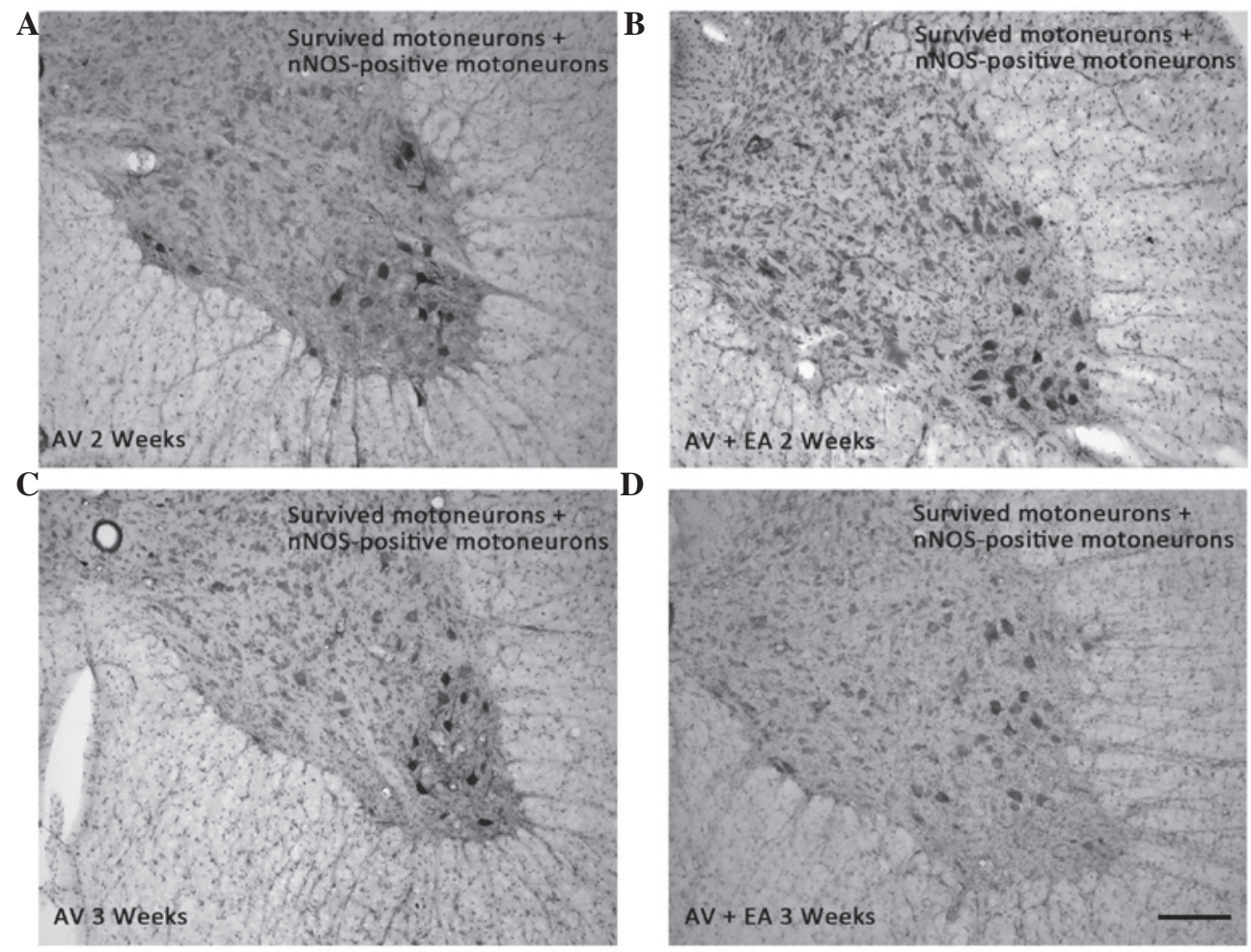

D

No. of survived motoneurons on lesion side

No. of motoneurons on contralateral side of the same section

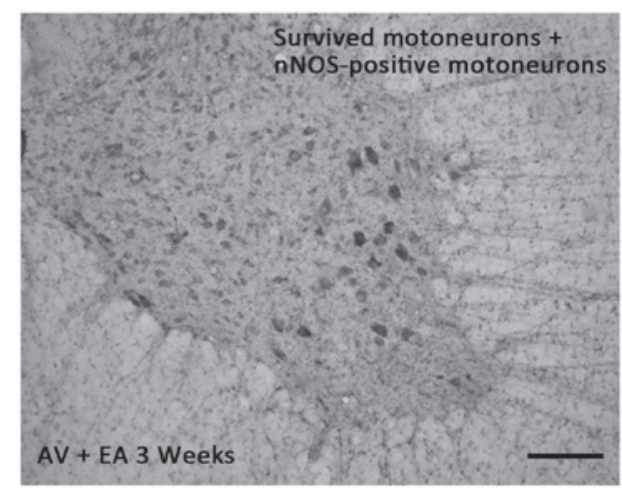

Figure 2. Effects of electroacupuncture (EA) on the expression of neuronal nitric oxide synthase (nNOS) and motoneuron survival. (A) Avulsion (AV) group, 2 weeks; (B) AV plus EA group, 2 weeks; (C) AV group, 3 weeks; (D) AV plus EA group, 3 weeks. Bar, $200 \mu \mathrm{m}$.

A

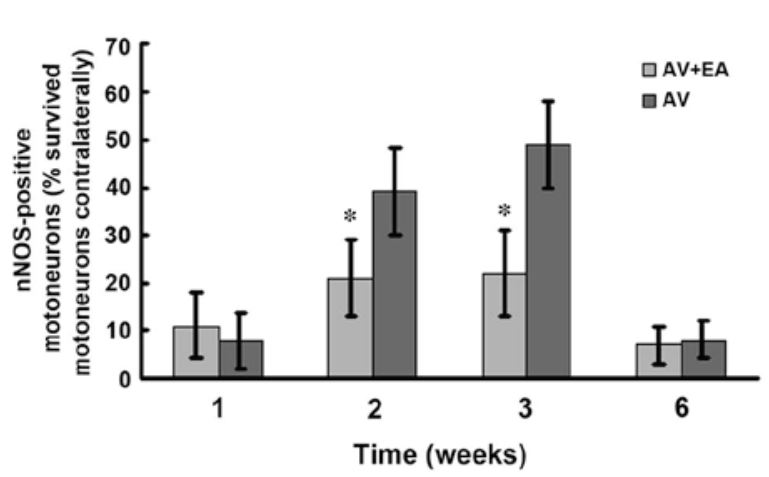

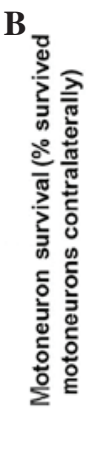

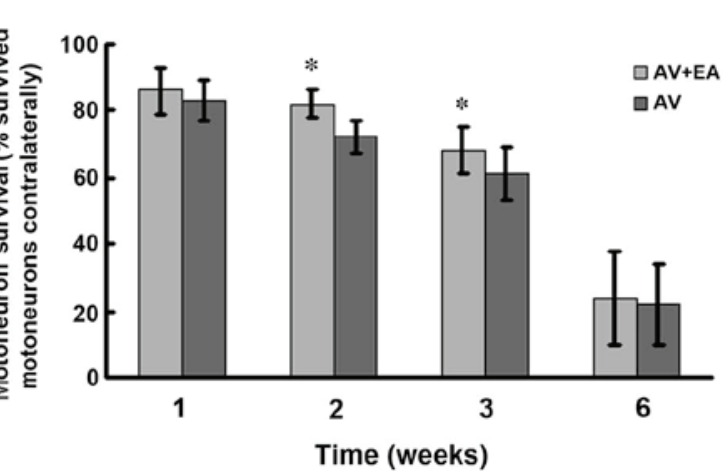

Figure 3. Statistical analysis of the effect of electroacupuncture (EA) on motoneurons following brachial plexus root avulsion. Data are expressed as the means $\pm \mathrm{SD}$ ( $n=5$ rats per group). The differences between the two groups were compared using the independent samples t-test or the Wilcoxon non-parametric test. ${ }^{*} \mathrm{P}<0.05$ vs. avulsion (AV) group at the same time point.

indepent samples t-test or a Wilcoxon non-parametric test if necessary. $\mathrm{P}<0.05$ was considered to indicate a statistically significant difference.

\section{Results}

EA at Dazhui (DU4) and Shousanli (LI10) decreases the 
number of nNOS-positive motoneurons following BPRA. The NADPH-d histochemistry stain revealed that BPRA caused the expression of nNOS in the motoneurons on the side of the lesion (Fig. 2). At 1, 2, 3 and 6 weeks, the percentages of nNOS-positive motoneurons in the two groups were $11 \pm 7$ vs. $8 \pm 6 \%$, $21 \pm 8$ vs. $39 \pm 9 \%, 22 \pm 9$ vs. $49 \pm 9 \%$ and $7 \pm 4$ vs. $8 \pm 4 \%$, respectively. In the EA+AV group, the percentage of nNOS-positive motoneurons was significantly lower compared to that in the $\mathrm{AV}$ group $(\mathrm{P}<0.05)$ at 2 and 3 weeks. However, there were no significant differences between the two groups at 1 and 6 weeks (Fig. 3).

EA at Dazhui (DU4) and Shousanli (LI10) increases the percentage of survived motoneurons following BPRA. The NR staining revealed that the BPRA caused loss of motoneurons in the ventral horns on the side of the lesion (Fig. 2). At 1 , 2,3 and 6 weeks, the percentages of survived motoneurons in the two groups were $86 \pm 7$ vs. $83 \pm 6 \%, 82 \pm 4$ vs. $72 \pm 5 \%$, $68 \pm 7$ vs. $61 \pm 8 \%$ and $24 \pm 14$ vs. $22 \pm 12 \%$, respectively. In the $\mathrm{EA}+\mathrm{AV}$ group, the percentage of survived motoneurons was significantly higher compared to that in the AV group $(\mathrm{P}<0.05)$ at 2 and 3 weeks. However, there were no significant differences between the two groups at 1 and 6 weeks (Fig. 3).

\section{Discussion}

This study demonstrated that EA stimulation at the acupoints of Dazhui (DU4) and Shousanli (LI10) was able to protect motoneurons during the early period after BPRA by reducing the expression of nNOS, which indicated that EA exerts a positive effect on PNI.

Previous studies have demonstrated that motoneuron death following BPRA was mainly due to the peroxidation mechanism and EA was able to reduce the oxidative stress through different ways in different models. In a rat model of smoke-induced chronic obstructive pulmonary disease (COPD), EA stimulation at Zusanli (ST36) reduced lung injury due to its anti-inflammatory and antioxidant effects $(27,28)$. In a rat model of vascular dementia, EA at related Jing-Well acupoints significantly improved memory impairment by decreasing the overproduction of $\mathrm{NO}$ and enhancing the ability of eliminating free radicals (29). In a rabbit model of hepatic ischemia-reperfusion injury, EA at Yanglingquan (GB34) inbibited the production of iNOS in the liver, reduced the content of nitric oxide (NO) and malondialdehyde (MDA) and increased the activity of superoxide dismutase (SOD) (30). Likewise, in a rat model of streptozocin-induced Alzheimer's disease, a PD rat model, a hypothalamus of spontaneously hypertensive rats model, a cerebral ischemia-reperfusion injury rat model and a rabbit model of knee osteoarthritis, EA played pivotal roles on reducing oxidative stress by raising the content of SOD, lowering MDA or NO and even changing the blood distribution and microcirculation of various organs (31-35). Notably, EA affected the levels of SOD and MDA in different organs in a hyperlipemia rat model (36). It was also demonstrated that the natural antioxidants-Egb-761 and TA9001 may protect motoneurons following BPRA (37). Based on the findings mentioned previously, we hypothesized that EA protected motoneurons by inhibiting the expression of nNOS due to its antioxidant effects.
It is generally accepted that neurotrophic factors may prevent motoneuron death following PNI (38-41). In the hippocampus, following cerebral ischemia-reperfusion injury in a mouse model, EA treatment enhanced endogenous BDNF expression, thereby possibly improving the survival environment for intracerebral neurons $(42,43)$. In a brachial plexus axotomy rabbit model, EA treatment increased the content of GDNF in the spinal cord root ganglia (44). Those findings demonstrated that EA was able to increase the content of neurotrophic factors, contributing to motoneuron regeneration.

However, our results demonstrated that EA exerted no significant effects on BPRA at 1 and 6 weeks. Due to the short treatment time from the completion of the operations for the model establishment to the sacrifice of the animals at the time point of 1 week, the effects of EA on inhibiting the expression of nNOS may not be obvious. Furthermore, the peak of motoneuron loss did not occur during the first week; thus, the number of survived motoneurons between the two groups may not be significantly different at this time point. Furthermore, at 6 weeks, EA was not shown to exert a protective effect against motoneuron death, mainly because BPRA is a severe injury which may lead to complete loss of motoneurons (1), despite several protective efforts. Therefore, EA as adjuvant therapy may effectively delay motoneuron death only when primary surgical treatment prevents complete motoneuron loss.

In this study, we first investigated the effects of EA on the BPRA model and demonstrated its efficiency, which may expand the application range of EA in different diseases. As a TCM therapy, EA may be a useful adjuvant therapy to surgery for BPRA and PNI.

Several related studies are currently in progress. In order to investigate whether the protection of motoneurons is achieved through the inhibition of nNOS, several experiments are required in the future. Firstly, the SOD, MDA and NO content must be assessed to verify that the decrease in nNOS expression is definitively caused by EA. Furthermore, their expression levels may be up- and downregulated to elucidate the mechanism underlying the action of EA. Finally, behavioral testing may be monitored to determine whether EA is able to promote sensory recovery.

\section{Acknowledgements}

This project was funded by grants from the National Natural Science Foundation of China (nos. 81070955, 31171290 and 81303115), the Natural Science Foundation of Guangdong Province (nos. S20134203030 and S2013040016915) and the Postdoctoral Foundation of China (no. BBK42913K09).

\section{References}

1. Matsuura Y, Iwakura N, Ohtori S, et al: The effect of anti-NGF receptor (p75 neurotrophin receptor) antibodies on nociceptive behavior and activation of spinal microglia in the rat brachial plexus avulsion model. Spine (Phila Pa 1976) 38: E332-E338, 2013.

2. Liu Z and Martin LJ: Motor neurons rapidly accumulate DNA single-strand breaks after in vitro exposure to nitric oxide and peroxynitrite and in vivo axotomy. J Comp Neurol 432: 35-60, 2001.

3. Martin LJ, Chen K and Liu Z: Adult motor neuron apoptosis is mediated by nitric oxide and Fas death receptor linked by DNA damage and p53 activation. J Neurosci 25: 6449-6459, 2005. 
4. Martin LJ, Kaiser A and Price AC: Motor neuron degeneration after sciatic nerve avulsion in adult rat evolves with oxidative stress and is apoptosis. J Neurobiol 40: 185-201, 1999.

5. Novikov L, Novikova L and Kellerth JO: Brain-derived neurotrophic factor promotes survival and blocks nitric oxide synthase expression in adult rat spinal motoneurons after ventral avulsion. Neurosci Lett 200: 45-48, 1995.

6. $\mathrm{Wu} \mathrm{W}$ : Potential roles of gene expression change in adult rat spinal motoneurons following axonal injury: a comparison among c-jun, off-affinity nerve growth factor receptor (LNGFR), and nitric oxide synthase (NOS). Exp Neurol 141: 190-200, 1996.

7. Zhou LH and Wu W: Antisense oligos to neuronal nitric oxide synthase aggravate motoneuron death induced by spinal root avulsion in adult rat. Exp Neurol 197: 84-92, 2006.

8. Zhou LH, Han S, Xie YY, et al: Differences in c-jun and nNOS expression levels in motoneurons following different kinds of axonal injury in adult rats. Brain Cell Biol 36: 213-227, 2008.

9. Chai H, Wu W, So KF and Yip HK: Survival and regeneration of motoneurons in adult rats by reimplantation of ventral root following spinal root avulsion. Neuroreport 11: 1249-1252, 2000

10. $\mathrm{Su} \mathrm{H}$, Chu TH and $\mathrm{Wu} \mathrm{W}$ : Lithium enhances proliferation and neuronal differentiation of neural progenitor cells in vitro and after transplantation into the adult rat spinal cord. Exp Neurol 206: 296-307, 2007

11. Hoang NS, Sar C, Valmier J, Sieso V and Scamps F: Electro-acupuncture on functional peripheral nerve regeneration in mice: a behavioural study. BMC Complement Altern Med 31: $141,2012$.

12. Hao J, Zhao C, Cao S, et al: Electric acupuncture treatment of peripheral nerve injury. J Tradit Chin Med 15: 114-117, 1995.

13. Chou WC, Liu HJ, Lin YW, et al: $2 \mathrm{~Hz}$ electro-acupuncture at yinlingquan (SP9) and ququan (LR8) acupoints induces changes in blood flow in the liver and spleen. Am J Chin Med 40: 75-84, 2012.

14. Li WJ, Pan SQ, Zeng YS, et al: Identification of acupuncturespecific proteins in the process of electro-acupuncture after spinal cord injury. Neurosci Res 67: 307-316, 2010.

15. Inoue $M$, Hojo $T$, Yano $T$ and Katsumi $Y$ : The effects of electroacupuncture on peripheral nerve regeneration in rats. Acupunct Med 21: 9-17, 2003.

16. Inoue M, Katsumi Y, Itoi M, et al: Direct current electrical stimulation of acupuncture needles for peripheral nerve regeneration: an exploratory case series. Acupunct Med 29: 88-93, 2011.

17. Pan P, Zhang X, Qian H, et al: Effects of electro-acupuncture on endothelium-derived endothelin-1 and endothelial nitric oxide synthase of rats with hypoxia-induced pulmonary hypertension. Exp Biol Med (Maywood) 235: 642-648, 2010.

18. Cha MH, Bai SJ, Lee KH, et al: Acute electroacupuncture inhibits nitric oxide synthase expression in the spinal cord of neuropathic rats. Neurol Res 32 (Suppl 1): 96-100, 2010.

19. Du J, Sun ZL, Jia J, et al: High-frequency electro-acupuncture stimulation modulates intracerebral $\gamma$-aminobutyric acid content in rat model of Parkinson's disease. Acta Physiol Sin 63: 305-310, 2011 (In Chinese)

20. Dai Y,Kondo E, Fukuoka T, et al: The effect of electroacupuncture on pain behaviors and noxious stimulus-evoked Fos expression in a rat model of neuropathic pain. J Pain 2: 151-159, 2001.

21. Wang Q: Acupuncture Therapeutics. 2nd edition. Chinese Medicine Press, Beijing, pp63-64, 2007.

22. $\mathrm{Li} \mathrm{Z}$ : International standardization of English translation of Chinese basic terminology of Chinese Medicine. 1st edition. Shanghai Science and Technology Press, Shanghai, pp285, 2008.

23. Wang H, Pan Y, Xue B, et al: The antioxidative effect of electro-acupuncture in a mouse model of Parkinson's disease. PLoS One 6: e19790, 2011.

24. Shen Y: Meridian, Collateral and Acupoints. 2nd edition. Chinese Medicine Press, Beijing, pp25-27, 2007.

25. Wang C: Basic skills of operating experimental animal in basic medicine. 1st edition. China Medical Science and Technology Press, Beijing, pp140-142, 2009.
26. Zhou LH and Wu W: Survival of injured spinal motoneurons in adult rat upon treatment with glial cell line-derived neurotrophic factor at 2 weeks but not at 4 weeks after root avulsion. J Neurotrauma 23: 920-927, 2006.

27. Geng WY, Liu ZB, Song NN, et al: Effects of electroacupuncture at Zusanli (ST36) on inflammatory cytokines in a rat model of smoke-induced chronic obstructive pulmonary disease. J Integr Med 11: 213-219, 2013.

28. Rho SW, Choi GS, Ko EJ, et al: Molecular changes in remote tissues induced by electro-acupuncture stimulation at acupoint ST36. Mol Cells 25: 178-183, 2008.

29. He F: Influences of electro-acupuncture at related jing-well points in rats with vascular dementia. J Tradit Chin Med 32: 238-242, 2012

30. Xie JP, Li W, Nong Y, et al: Effects of electroacupuncture at Fenglong (ST 40) on SOD and MDA in different organs of the hyperlipemia rat. Chin Acupunct Moxibust 28: 293-296, 2008 (In Chinese).

31. Zhou L, Li F, Yuan QF, et al: The comparative study of protective effect of two kinds of natural antioxidants on spinal motoneurons for lowing brachial roots avulsion. Chin J Microsurg 26: 204-206, 2003 (In Chinese).

32. Kim JI, Kim YS, Kang SK, et al: Electroacupuncture decreases nitric oxide synthesis in the hypothalamus of spontaneously hypertensive rats. Neurosci Lett 446: 78-82, 2008.

33. Li ZR, Shen MH and Niu WM: Involvement of melatonin in the adjusting effect of electroacupuncture in resisting oxygen stress in cerebral ischemia-reperfusion injury rats. Acupuncture Research 33: 164-168, 2008 (In Chinese).

34. Hsiao SH and Tsai LJ: A neurovascular transmission model for acupuncture-induced nitric oxide. J Acupunct Meridian Stud 1: 42-50, 2008.

35. Huang J, Zhuo LS, Wang YY and Feng WQ: Effects of electroacupuncture on synovia free radicals in rabbits with knee osteoarthritis. Acupuncture Research 33: 116-119, 2008 (In Chinese).

36. Zhou J and Chen Y: Effect of electroacupuncture preconditioning on nitric oxide, nitric oxide synthase and oxyradical during hepatic ischemia-reperfusion injury in rabbits. Chin Acupunct Moxibust 31: 149-152, 2011 (In Chinese).

37. Zhang P, Guan SS and Jiang GH: Effects of electroacupuncture on expression of Abeta positive cells of the hippocampus and SOD activity in rats with streptozocin-Alzheimer's disease. Chin Acupunct Moxibust 30: 1007-1010, 2010 (In Chinese).

38. Yuan Q, Wu W, So KF, et al: Effects of neurotrophic factors on motoneuron survival following axonal injury in newborn rats, MOTOR SYSTEMS. Neuroreport 11: 2237-2241, 2000.

39. Aloe L and Manni L: Low-frequency electro-acupuncture reduces the nociceptive response and the pain mediator enhancement induced by nerve growth factor. Neurosci Lett 449: 173-177, 2009.

40. Dong Z, Sun Y, Lu P, et al: Electroacupuncture and lumbar transplant of GDNF-secreting fibroblasts synergistically attenuate hyperalgesia after sciatic nerve constriction. Am J Chin Med 41: 459-472, 2013.

41. Liu Z, Ding Y and Zeng YS: A new combined therapeutic strategy of governor vessel electro-acupuncture and adult stem cell transplantation promotes the recovery of injured spinal cord. Curr Med Chem 18: 5165-5171, 2011.

42. Zhao J, Xu H, Tian Y, et al: Effect of electroacupuncture on brain-derived neurotrophic factor mRNA expression in mouse hippocampus following cerebral ischemia-reperfusion injury. J Tradit Chin Med 33: 253-257, 2013.

43. Kim MW, Chung YC, Jung HC, et al: Electroacupuncture enhances motor recovery performance with brain-derived neurotrophic factor expression in rats with cerebral infarction. Acupunct Med 30: 222-226, 2012.

44. Wang RH, Chen YF and Qu HY: The effects of needling on glia-derived neurotrophic factor (GDNF) after brachial plexus injury. J Shanxi College Tradit Chin Med 32: 66-68, 2009 (In Chinese) 